\title{
ON WELL-POSEDNESS ASSOCIATED WITH A CLASS OF CONTROLLED VARIATIONAL INEQUALITIES
}

\author{
Savin Treanţă $\breve{A}^{1, *} \odot$ and Shalini Jha ${ }^{2}$
}

\begin{abstract}
In this paper, by using the new concepts of monotonicity, pseudomonotonicity and hemicontinuity associated with the considered curvilinear integral functional, we investigate the wellposedness and well-posedness in generalized sense for a class of controlled variational inequality problems. More precisely, by introducing the approximating solution set of the considered class of controlled variational inequality problems, we formulate and prove some characterization results on well-posedness and well-posedness in generalized sense. Also, the theoretical developments presented in the paper are accompanied by illustrative examples.
\end{abstract}

Mathematics Subject Classification. 49K40,65K10.

Received March 15, 2021. Accepted August 31, 2021.

\section{INTRODUCTION}

The concept of well-posedness has significant role in the stability theory of optimization problems, which has been discussed in different areas of optimization likewise calculus of variations, mathematical programming, and optimal control. This notion is important for problems due to certainty of the existence of the solution. More precisely, for instance, a problem in differential equations is said to be well-posed if: (1) a solution exists; (2) that solution is unique; (3) the solution's behaviour changes continuously with the initial conditions. The first condition is almost trivial; it merely reflects the fact that it is possible to pose problems of differential equations in such a way that no solutions exist. Naturally, one would like at least one solution to their problem to exist. The second condition is a bit more interesting. Differential equations which satisfy criterion (1) will in general have many, in fact infinitely many, solution functions. Ensuring the uniqueness of solution to a problem becomes much more difficult for problems involving higher orders of derivatives, and even more so for PDEs. Criterion (3) holds if, when you make a "small" change to the initial or boundary conditions, your solutions changes only by a "small" amount. One may now begin to see the importance of well-posedness of a mathematical problem and the implications to physical prediction.

There are several methods to solve the optimization problems. Sometimes, it is very difficult to find out the solution by using these methods which may or may not ensures the exactness of the solutions. In these circumstances, the well-posedness of an optimization problem is important because it ensures the convergence

Keywords and phrases: Well-posedness, well-posedness in generalized sense, controlled variational inequality, monotonicity, pseudomonotonicity, hemicontinuity, curvilinear integral functional.

1 Department of Applied Mathematics, University Politehnica of Bucharest, 313 Splaiul Independenţei, Bucharest 060042, Romania.

2 School of Advanced Sciences, VIT-AP University, Andhra Pradesh 522237, India.

* Corresponding author: savin_treanta@yahoo.com 
of the sequence of approximate solutions obtained through iterative techniques. Tykhonov [30] first investigated the general concept of well-posedness for unconstrained minimization problems that requires the existence and uniqueness of minimizer and convergence of every minimizing sequence towards the unique minimizer. It is clear that the concept of well-posedness is motivated by the numerical methods producing optimizing sequences for optimization problems. Following the concept of Tykhonov well-posedness, various kinds of well-posedness for optimization problems, such as Levitin-Polyak well-posedness [16] and extended well-posedness, have been introduced and studied by many researchers (see $[2,4,14,17,18]$ ).

In the literature, several researchers studied the variational inequality over set valued mappings (see, for example, [31]). Under mild conditions, the variational inequality problems are closely related to the optimization problems, so the concept of Tykhonov well-posedness has also been extended to variational inequalities $[5,9,11,34]$, and thereafter, to some other problems like fixed point problems [7], hemivariational inequality problems [1, 21, 33, 34], equilibrium problems [6], complementary problems [10], and Nash equilibrium problems [19]. Ceng and Yao [3] discussed well-posedness of mixed variational inequality in generalized sense and proved that the well-posed generalized mixed variational inequality is equivalent to that of fixed point problems and inclusion problems. Thereafter, Huang et al. [12] investigated Levitin-Polyak generalized well-posedness for variational inequality problem and established some characterizations for the well-posednesses of constrained variational inequalities. Also, in [20], Lin and Chuang proposed generalized well-posedness for variational disclusion problems, inclusion problems and the minimization problems involving variational disclusion problems, inclusion problems as constraints. Later on, Lalita and Bhatia [15] proposed the well-posedness and generalized well-posedness for parametric type quasi-variational inequality problems and minimization problems, and discussed the cases in which the problem has one or more than one solution. Moreover, Fang et al. [8] extended this notion of well-posedness by applying perturbations on the mixed variational inequality problem over Banach space and proved various results for well-posedness via perturbations. Virmani and Srivastava [32] introduced the necessary and sufficient conditions for $\alpha, \alpha-L$ well-posedness and generalized $\alpha, \alpha-L$ well-posedness for a mixed vector quasi-variational-like inequality using bifunctions. Recently, Jayswal and Shalini [13] derived the well-posedness for generalized mixed vector variational-like inequality and optimization problems including this inequality as a constraint.

Moreover, the multi-time (multidimensional) control theory comes from calculus of variations which is applied to solve various operations research problems involving applied science and technology. In the last few years, it has been intensively studied in applied and theoretical points of view (see [22-26]). Multi-time variational inequality problem is another interesting generalization of variational inequality (see [27, 28]). In [29], Treanţă has established the weak sharp solutions for a variational-type inequality by defining $(\rho, b, d)$ convex path-independent curvilinear integral functional. Also, he studied an equivalence between the minimum principle sufficiency property and the weak sharpness property of the solution set associated with the considered variational-type inequality.

Inspired and motivated by the above research works, in the current paper, we investigate the well-posedness and well-posedness in generalized sense for a class of controlled variational inequality problems. More precisely, by using the new concepts of monotonicity, pseudomonotonicity and hemicontinuity associated with the considered curvilinear integral functional, and the approximating solution set of the considered class of controlled variational inequality problems, we formulate and prove some characterization results on well-posedness and well-posedness in generalized sense. The presence of the curvilinear integral functionals and of the mathematical framework governed by infinite-dimensional function spaces, represent the main novelty elements of this paper (the former works are studied in the classical finite-dimensional spaces). Furthermore, besides totally new elements mentioned above, due to the physical meaning of the integral functionals used (as is well-known the path-independent curvilinear integrals represent the mechanical work performed by a variable force in order to move its point of application along a given piecewise smooth curve), this paper becomes a fundamental work for researchers in the field of applied mathematics and ingineering.

The paper is organized as follows. The new notions of monotonicity, pseudomonotonicity and hemicontinuity associated with a curvilinear integral functional, and an auxiliary lemma are provided in Section 2. In Section 3, 
we investigate about well-posedness and well-posedness in generalized sense, by considering the approximating solution set of the considered class of controlled variational inequality problems. Further, under suitable hypotheses, we establish that well-posedness is characterized in the terms of existence and uniqueness of solution. Also, we formulate and prove sufficient conditions for the well-posedness in generalized sense by assuming the boundedness of approximate solutions. In order to illustrate the theoretical results derived in this paper, we also provide some examples. Section 4 concludes the paper.

\section{NOTATIONS AND PRELIMINARIES}

In this paper, we consider the following notations and mathematical tools. Denote by $\Omega$ a compact domain in $\mathbb{R}^{m}$ and consider the point $\Omega \ni s=\left(s^{\nu}\right), \nu=\overline{1, m}$, as a multi-parameter of evolution. Let $\Omega \supset \Upsilon: s=(s(\varsigma)), \varsigma \in$ $[a, b]$ (or $s \in \overline{s_{1}, s_{2}}$ ) be a piecewise smooth curve joining two different points $s_{1}=\left(s_{1}^{1}, \ldots, s_{1}^{m}\right), s_{2}=\left(s_{2}^{1}, \ldots, s_{2}^{m}\right)$ in $\Omega$. Consider $\mathcal{X}$ as the space of all piecewise smooth state functions $a: \Omega \rightarrow \mathbb{R}^{n}$, equipped with the norm

$$
\|a\|=\|a\|_{\infty}+\sum_{\nu=1}^{m}\left\|a_{\nu}\right\|_{\infty}, \quad \forall a \in \mathcal{X},
$$

where we used the notation $a_{\nu}:=\frac{\partial a}{\partial s^{\nu}}, \nu=\overline{1, m}$, and let $\mathcal{U}$ be the space of piecewise continuous control functions $u: \Omega \rightarrow \mathbb{R}^{k}$, endowed with the uniform norm $\|\cdot\|_{\infty}$.

Throughout this paper, we consider that $X \times U$ is a nonempty, closed and convex subset of $\mathcal{X} \times \mathcal{U}$, equipped with the inner product

$$
\begin{gathered}
\langle(a, u),(b, w)\rangle=\int_{\Upsilon}[a(s) \cdot b(s)+u(s) \cdot w(s)] \mathrm{d} s^{\nu} \\
=\int_{\Upsilon}\left[\sum_{i=1}^{n} a^{i}(s) b^{i}(s)+\sum_{j=1}^{k} u^{j}(s) w^{j}(s)\right] \mathrm{d} s^{\nu} \\
=\int_{\Upsilon}\left[\sum_{i=1}^{n} a^{i}(s) b^{i}(s)+\sum_{j=1}^{k} u^{j}(s) w^{j}(s)\right] \mathrm{d} s^{1}+\cdots+\left[\sum_{i=1}^{n} a^{i}(s) b^{i}(s)+\sum_{j=1}^{k} u^{j}(s) w^{j}(s)\right] \mathrm{d} s^{m}, \\
\forall(a, u),(b, w) \in \mathcal{X} \times \mathcal{U}
\end{gathered}
$$

and the induced norm.

Let $J^{1}\left(\mathbb{R}^{m}, \mathbb{R}^{n}\right)$ be the jet bundle of first-order associated with $\mathbb{R}^{m}$ and $\mathbb{R}^{n}$. By using the Lagrange 1-form density $f_{\nu}: J^{1}\left(\mathbb{R}^{m}, \mathbb{R}^{n}\right) \times \mathbb{R}^{k} \rightarrow \mathbb{R}, \nu=\overline{1, m}$, we define the following functional governed by a curvilinear integral (see summation over the repeated indices, Einstein summation):

$$
\begin{gathered}
F: \mathcal{X} \times \mathcal{U} \rightarrow \mathbb{R}, \quad F(a, u)=\int_{\Upsilon} f_{\nu}\left(s, a(s), a_{\kappa}(s), u(s)\right) \mathrm{d} s^{\nu} \\
=\int_{\Upsilon} f_{1}\left(s, a(s), a_{\kappa}(s), u(s)\right) \mathrm{d} s^{1}+\cdots+f_{m}\left(s, a(s), a_{\kappa}(s), u(s)\right) \mathrm{d} s^{m},
\end{gathered}
$$

where $a_{\kappa}(s)=\frac{\partial a}{\partial s^{\kappa}}(s), \kappa \in\{1, \ldots, m\}$, denotes the partial velocities.

By using the above mathematical tools, we introduce the following controlled variational inequality problem (for short, CVIP), formulated as: find $(a, u) \in X \times U$ such that

$$
\int_{\Upsilon}\left[\frac{\partial f_{\nu}}{\partial a}\left(\psi_{a, u}(s)\right)(b(s)-a(s))+\frac{\partial f_{\nu}}{\partial a_{\kappa}}\left(\psi_{a, u}(s)\right) D_{\kappa}(b(s)-a(s))\right] \mathrm{d} s^{\nu}
$$




$$
+\int_{\Upsilon}\left[\frac{\partial f_{\nu}}{\partial u}\left(\psi_{a, u}(s)\right)(w(s)-u(s))\right] \mathrm{d} s^{\nu} \geq 0, \quad \forall(b, w) \in X \times U,
$$

where $D_{\kappa}:=\frac{\partial}{\partial s^{\kappa}}$ is the total derivative operator and $\left(\psi_{a, u}(s)\right):=\left(s, a(s), a_{\kappa}(s), u(s)\right)$.

Let $\Theta$ be the set of all feasible solutions of (CVIP), that is,

$$
\begin{aligned}
& \Theta=\left\{(a, u) \in X \times U: \int_{\Upsilon}[\right.(b(s)-a(s)) \frac{\partial f_{\nu}}{\partial a}\left(\psi_{a, u}(s)\right) \\
&+ D_{\kappa}(b(s)-a(s)) \frac{\partial f_{\nu}}{\partial a_{\kappa}}\left(\psi_{a, u}(s)\right) \\
&+\left.(w(s)-u(s)) \frac{\partial f_{\nu}}{\partial u}\left(\psi_{a, u}(s)\right)\right] \mathrm{d} s^{\nu} \geq 0, \\
&\forall(b, w) \in X \times U\} .
\end{aligned}
$$

Further, by considering the notion of monotonicity for variational inequality problems, we introduce the concepts of monotonicity and pseudomonotonicity associated with the aforementioned curvilinear integral functional.

Definition 2.1. The curvilinear integral functional $F$ is called monotone on $X \times U$ if the following inequality holds:

$$
\begin{gathered}
\int_{\Upsilon}\left[(a(s)-b(s))\left(\frac{\partial f_{\nu}}{\partial a}\left(\psi_{a, u}(s)\right)-\frac{\partial f_{\nu}}{\partial a}\left(\psi_{b, w}(s)\right)\right)\right. \\
+(u(s)-w(s))\left(\frac{\partial f_{\nu}}{\partial u}\left(\psi_{a, u}(s)\right)-\frac{\partial f_{\nu}}{\partial u}\left(\psi_{b, w}(s)\right)\right) \\
\left.+D_{\kappa}(a(s)-b(s))\left(\frac{\partial f_{\nu}}{\partial a_{\kappa}}\left(\psi_{a, u}(s)\right)-\frac{\partial f_{\nu}}{\partial a_{\kappa}}\left(\psi_{b, w}(s)\right)\right)\right] \mathrm{d} s^{\nu} \geq 0 \\
\forall(a, u),(b, w) \in X \times U .
\end{gathered}
$$

Example 2.2. Let $\nu \in\{1,2\}, \Omega=[0,1]^{2}$ and $\Omega \supset \Upsilon$ be a piecewise smooth curve joining the points $(0,0),(1,1)$ in $\Omega$. Consider

$$
\begin{gathered}
f_{\nu}\left(\psi_{a, u}(s)\right) \mathrm{d} s^{\nu}=f_{1}\left(\psi_{a, u}(s)\right) \mathrm{d} s^{1}+f_{2}\left(\psi_{a, u}(s)\right) \mathrm{d} s^{2} \\
=\left[u(s)+\frac{\partial a}{\partial s^{1}}+\frac{\partial a}{\partial s^{2}}\right] \mathrm{d} s^{1}+\left(e^{a(s)}-1\right) \mathrm{d} s^{2} .
\end{gathered}
$$

Now, we show that the curvilinear integral functional $\int_{\Upsilon} f_{\nu}\left(\psi_{a, u}(s)\right) \mathrm{d} s^{\nu}$ is monotone on $X \times U=C^{1}(\Omega, \mathbb{R}) \times$ $C(\Omega, \mathbb{R})$. Indeed, we have

$$
\begin{aligned}
\int_{\Upsilon}[(a(s)-b(s))( & \left.\frac{\partial f_{\nu}}{\partial a}\left(\psi_{a, u}(s)\right)-\frac{\partial f_{\nu}}{\partial a}\left(\psi_{b, w}(s)\right)\right) \\
& +(u(s)-w(s))\left(\frac{\partial f_{\nu}}{\partial u}\left(\psi_{a, u}(s)\right)-\frac{\partial f_{\nu}}{\partial u}\left(\psi_{b, w}(s)\right)\right) \\
& \left.+D_{\kappa}(a(s)-b(s))\left(\frac{\partial f_{\nu}}{\partial a_{\kappa}}\left(\psi_{a, u}(s)\right)-\frac{\partial f_{\nu}}{\partial a_{\kappa}}\left(\psi_{b, w}(s)\right)\right)\right] \mathrm{d} s^{\nu}
\end{aligned}
$$




$$
=\int_{\Upsilon}(a(s)-b(s))\left(e^{a(s)}-e^{b(s)}\right) \mathrm{d} s^{2} \geq 0, \forall(a, u),(b, w) \in X \times U .
$$

Definition 2.3. The curvilinear integral functional $F$ is called pseudomonotone on $X \times U$ if the following implication holds:

$$
\begin{gathered}
\int_{\Upsilon}\left[(a(s)-b(s)) \frac{\partial f_{\nu}}{\partial a}\left(\psi_{b, w}(s)\right)+(u(s)-w(s)) \frac{\partial f_{\nu}}{\partial u}\left(\psi_{b, w}(s)\right)\right. \\
\left.+D_{\kappa}(a(s)-b(s)) \frac{\partial f_{\nu}}{\partial a_{\kappa}}\left(\psi_{b, w}(s)\right)\right] \mathrm{d} s^{\nu} \geq 0 \\
\Rightarrow \int_{\Upsilon}\left[(a(s)-b(s)) \frac{\partial f_{\nu}}{\partial a}\left(\psi_{a, u}(s)\right)+(u(s)-w(s)) \frac{\partial f_{\nu}}{\partial u}\left(\psi_{a, u}(s)\right)\right. \\
\left.+D_{\kappa}(a(s)-b(s)) \frac{\partial f_{\nu}}{\partial a_{\kappa}}\left(\psi_{a, u}(s)\right)\right] \mathrm{d} s^{\nu} \geq 0, \\
\forall(a, u),(b, w) \in X \times U .
\end{gathered}
$$

In the following, we present an example of curvilinear integral functional which is pseudomonotone but not monotone.

Example 2.4. Let $\nu \in\{1,2\}, \Omega=[0,1]^{2}$ and $\Omega \supset \Upsilon$ be a piecewise smooth curve joining the points $(0,0),(1,1)$ in $\Omega$. Consider

$$
\begin{gathered}
f_{\nu}\left(\psi_{a, u}(s)\right) \mathrm{d} s^{\nu}=f_{1}\left(\psi_{a, u}(s)\right) \mathrm{d} s^{1}+f_{2}\left(\psi_{a, u}(s)\right) \mathrm{d} s^{2} \\
=\left[\frac{\partial a}{\partial s^{1}}+\sin u(s)\right] \mathrm{d} s^{1}+a(s) e^{a(s)} \mathrm{d} s^{2}
\end{gathered}
$$

Now, we show that the curvilinear integral functional $\int_{\Upsilon} f_{\nu}\left(\psi_{a, u}(s)\right) \mathrm{d} s^{\nu}$ is pseudomonotone on $X \times U=$ $C^{1}(\Omega,[-1,1]) \times C(\Omega,[-1,1])$. Indeed, we have

$$
\begin{gathered}
\int_{\Upsilon}\left[(a(s)-b(s)) \frac{\partial f_{\nu}}{\partial a}\left(\psi_{b, w}(s)\right)+(u(s)-w(s)) \frac{\partial f_{\nu}}{\partial u}\left(\psi_{b, w}(s)\right)\right. \\
\left.+D_{\kappa}(a(s)-b(s)) \frac{\partial f_{\nu}}{\partial a_{\kappa}}\left(\psi_{b, w}(s)\right)\right] \mathrm{d} s^{\nu} \\
=\int_{\Upsilon}\left[(u(s)-w(s)) \cos w(s)+D_{1}(a(s)-b(s))\right] \mathrm{d} s^{1}+(a(s)-b(s))\left(e^{b(s)}+b(s) e^{b(s)}\right) \mathrm{d} s^{2} \geq 0 \\
\forall(a, u),(b, w) \in X \times U \\
\Rightarrow \int_{\Upsilon}\left[(a(s)-b(s)) \frac{\partial f_{\nu}}{\partial a}\left(\psi_{a, u}(s)\right)+(u(s)-w(s)) \frac{\partial f_{\nu}}{\partial u}\left(\psi_{a, u}(s)\right)\right. \\
\left.+D_{\kappa}(a(s)-b(s)) \frac{\partial f_{\nu}}{\partial a_{\kappa}}\left(\psi_{a, u}(s)\right)\right] \mathrm{d} s^{\nu} \\
=\int_{\Upsilon}\left[(u(s)-w(s)) \cos u(s)+D_{1}(a(s)-b(s))\right] \mathrm{d} s^{1}+(a(s)-b(s))\left(e^{a(s)}+a(s) e^{a(s)}\right) \mathrm{d} s^{2} \geq 0
\end{gathered}
$$




$$
\forall(a, u),(b, w) \in X \times U \text {. }
$$

But it is not monotone on $X \times U$, because

$$
\begin{aligned}
& \int_{\Upsilon}[(a(s)-b(s))(\left.\frac{\partial f_{\nu}}{\partial a}\left(\psi_{a, u}(s)\right)-\frac{\partial f_{\nu}}{\partial a}\left(\psi_{b, w}(s)\right)\right) \\
&+(u(s)-w(s))\left(\frac{\partial f_{\nu}}{\partial u}\left(\psi_{a, u}(s)\right)-\frac{\partial f_{\nu}}{\partial u}\left(\psi_{b, w}(s)\right)\right) \\
&\left.+D_{\kappa}(a(s)-b(s))\left(\frac{\partial f_{\nu}}{\partial a_{\kappa}}\left(\psi_{a, u}(s)\right)-\frac{\partial f_{\nu}}{\partial a_{\kappa}}\left(\psi_{b, w}(s)\right)\right)\right] \mathrm{d} s^{\nu} \\
&=\int_{\Upsilon}(u(s)-w(s))(\cos u(s)-\cos w(s)) \mathrm{d} s^{1} \\
&+(a(s)-b(s))\left(a(s) e^{a(s)}+e^{a(s)}-b(s) e^{b(s)}-e^{b(s)}\right) \mathrm{d} s^{2} \ngtr 0, \\
& \forall(a, u),(b, w) \in X \times U .
\end{aligned}
$$

Assumption. In this paper, we assume the following working hypothesis:

$$
d G:=D_{\kappa}\left[\frac{\partial f_{\nu}}{\partial a_{\kappa}}(a-b)\right] \mathrm{d} s^{\nu}
$$

is an exact total differential satisfying $G\left(s_{1}\right)=G\left(s_{2}\right)$.

Inspired by Usman and Khan [31], we introduce the following definition of hemicontinuity for the aforementioned curvilinear integral functional.

Definition 2.5. The curvilinear integral functional $F$ is said to be hemicontinuous on $X \times U$ if, for $\forall(a(s), u(s)),(b(s), w(s)) \in X \times U$, the application

$$
\lambda \rightarrow\left\langle\left((a(s), u(s))-(b(s), w(s)),\left(\frac{\delta_{\nu} F}{\delta a_{\lambda}}, \frac{\delta_{\nu} F}{\delta u_{\lambda}}\right)\right\rangle, \quad 0 \leq \lambda \leq 1\right.
$$

is continuous at $0^{+}$, where

$$
\begin{gathered}
\frac{\delta_{\nu} F}{\delta a_{\lambda}}:=\frac{\partial f_{\nu}}{\partial a}\left(\psi_{a_{\lambda}, u_{\lambda}}(s)\right)-D_{\kappa} \frac{\partial f_{\nu}}{\partial a_{\kappa}}\left(\psi_{a_{\lambda}, u_{\lambda}}(s)\right) \in X, \\
\frac{\delta_{\nu} F}{\delta u_{\lambda}}:=\frac{\partial f_{\nu}}{\partial u}\left(\psi_{a_{\lambda}, u_{\lambda}}(s)\right) \in U, \\
a_{\lambda}:=\lambda a+(1-\lambda) b, \quad u_{\lambda}:=\lambda u+(1-\lambda) w .
\end{gathered}
$$

The following lemma is an auxiliary result for proving the main results derived in the present paper.

Lemma 2.6. Consider the curvilinear integral functional $F$ is pseudomonotone and hemicontinuous on $X \times U$. A pair $(a, u) \in X \times U$ is solution of $(C V I P)$ if and only if it is solution for

$$
\begin{aligned}
& \int_{\Upsilon}\left[(b(s)-a(s)) \frac{\partial f_{\nu}}{\partial a}\left(\psi_{b, w}(s)\right)+(w(s)-u(s)) \frac{\partial f_{\nu}}{\partial u}\left(\psi_{b, w}(s)\right)\right. \\
& \left.+D_{\kappa}(b(s)-a(s)) \frac{\partial f_{\nu}}{\partial a_{\kappa}}\left(\psi_{b, w}(s)\right)\right] \mathrm{d} s^{\nu} \geq 0, \quad \forall(b, w) \in X \times U .
\end{aligned}
$$


Proof. Firstly, consider the pair $(a, u) \in X \times U$ solves (CVIP), that is,

$$
\begin{aligned}
& \int_{\Upsilon}\left[(b(s)-a(s)) \frac{\partial f_{\nu}}{\partial a}\left(\psi_{a, u}(s)\right)+(w(s)-u(s)) \frac{\partial f_{\nu}}{\partial u}\left(\psi_{a, u}(s)\right)\right. \\
& \left.+D_{\kappa}(b(s)-a(s)) \frac{\partial f_{\nu}}{\partial a_{\kappa}}\left(\psi_{a, u}(s)\right)\right] \mathrm{d} s^{\nu} \geq 0, \quad \forall(b, w) \in X \times U .
\end{aligned}
$$

By using the definition of pseudomonotonicity, the above inequality implies

$$
\begin{aligned}
& \int_{\Upsilon}\left[(b(s)-a(s)) \frac{\partial f_{\nu}}{\partial a}\left(\psi_{b, w}(s)\right)+(w(s)-u(s)) \frac{\partial f_{\nu}}{\partial u}\left(\psi_{b, w}(s)\right)\right. \\
& \left.+D_{\kappa}(b(s)-a(s)) \frac{\partial f_{\nu}}{\partial a_{\kappa}}\left(\psi_{b, w}(s)\right)\right] \mathrm{d} s^{\nu} \geq 0, \quad \forall(b, w) \in X \times U .
\end{aligned}
$$

Conversely, assume that

$$
\begin{aligned}
& \int_{\Upsilon}\left[(b(s)-a(s)) \frac{\partial f_{\nu}}{\partial a}\left(\psi_{b, w}(s)\right)+(w(s)-u(s)) \frac{\partial f_{\nu}}{\partial u}\left(\psi_{b, w}(s)\right)\right. \\
& \left.+D_{\kappa}(b(s)-a(s)) \frac{\partial f_{\nu}}{\partial a_{\kappa}}\left(\psi_{b, w}(s)\right)\right] \mathrm{d} s^{\nu} \geq 0, \quad \forall(b, w) \in X \times U .
\end{aligned}
$$

Now, for $(b, w) \in X \times U$ and $\lambda \in(0,1]$, define

$$
\left(b_{\lambda}, w_{\lambda}\right)=((1-\lambda) a+\lambda b,(1-\lambda) u+\lambda w) \in X \times U
$$

Thus, the above inequality can be rewritten as

$$
\begin{gathered}
\int_{\Upsilon}\left[\left(b_{\lambda}(s)-a(s)\right) \frac{\partial f_{\nu}}{\partial a}\left(\psi_{b_{\lambda}, w_{\lambda}}(s)\right)+\left(w_{\lambda}(s)-u(s)\right) \frac{\partial f_{\nu}}{\partial u}\left(\psi_{b_{\lambda}, w_{\lambda}}(s)\right)\right. \\
\left.+D_{\kappa}\left(b_{\lambda}(s)-a(s)\right) \frac{\partial f_{\nu}}{\partial a_{\kappa}}\left(\psi_{b_{\lambda}, w_{\lambda}}(s)\right)\right] \mathrm{d} s^{\nu} \geq 0, \quad(b, w) \in X \times U .
\end{gathered}
$$

Taking $\lambda \rightarrow 0$ and using the hemicontinuity property associated with the considered curvilinear integral functional, we have

$$
\begin{aligned}
& \int_{\Upsilon}\left[(b(s)-a(s)) \frac{\partial f_{\nu}}{\partial a}\left(\psi_{a, u}(s)\right)+(w(s)-u(s)) \frac{\partial f_{\nu}}{\partial u}\left(\psi_{a, u}(s)\right)\right. \\
& \left.+D_{\kappa}(b(s)-a(s)) \frac{\partial f_{\nu}}{\partial a_{\kappa}}\left(\psi_{a, u}(s)\right)\right] \mathrm{d} s^{\nu} \geq 0, \quad \forall(b, w) \in X \times U,
\end{aligned}
$$

which shows that $(a, u)$ solves (CVIP).

\section{WELL-POSEDNESS AND WELL-POSEDNESS IN GENERALIZED SENSE ASSOCIATED WITH (CVIP)}

In this section, by using the notions of monotonicity and hemicontinuity introduced in Section 2, we study the well-posedness and well-posedness in generalized sense of the considered class of controlled variational inequality problems. For this purpose, we introduce the following definitions. 
Definition 3.1. The sequence $\left\{\left(a_{n}, u_{n}\right)\right\} \in X \times U$ is called an approximating sequence of (CVIP) if there exists a sequence of positive real numbers $\iota_{n} \rightarrow 0$ as $n \rightarrow \infty$, such that the following inequality

$$
\begin{aligned}
& \int_{\Upsilon}\left[\left(b(s)-a_{n}(s)\right) \frac{\partial f_{\nu}}{\partial a}\left(\psi_{a_{n}, u_{n}}(s)\right)+\left(w(s)-u_{n}(s)\right) \frac{\partial f_{\nu}}{\partial u}\left(\psi_{a_{n}, u_{n}}(s)\right)\right. \\
& \left.+D_{\kappa}\left(b(s)-a_{n}(s)\right) \frac{\partial f_{\nu}}{\partial a_{\kappa}}\left(\psi_{a_{n}, u_{n}}(s)\right)\right] \mathrm{d} s^{\nu}+\iota_{n} \geq 0, \quad \forall(b, w) \in X \times U
\end{aligned}
$$

holds.

Definition 3.2. The problem (CVIP) is called well-posed if it satisfies the following two conditions:

(i) it possesses a single solution $\left(a_{0}, u_{0}\right)$;

(ii) each approximating sequence of (CVIP) will converge to this single solution $\left(a_{0}, u_{0}\right)$.

Definition 3.3. The problem (CVIP) is called well-posed in generalized sense if it satisfies the following two conditions:

(i) $\Theta \neq \emptyset$;

(ii) each approximating sequence of (CVIP) possesses a subsequence that will converge to some pair of $\Theta$.

In order to investigate the well-posedness and well-posedness in generalized sense of (CVIP), we introduce the definition of approximating solution set of (CVIP) as follows:

$$
\begin{aligned}
\Theta_{\iota}=\{(a, u) \in & X \times U: \int_{\Upsilon}\left[(b(s)-a(s)) \frac{\partial f_{\nu}}{\partial a}\left(\psi_{a, u}(s)\right)+(w(s)-u(s)) \frac{\partial f_{\nu}}{\partial u}\left(\psi_{a, u}(s)\right)\right. \\
& \left.\left.+D_{\kappa}(b(s)-a(s)) \frac{\partial f_{\nu}}{\partial a_{\kappa}}\left(\psi_{a, u}(s)\right)\right] \mathrm{d} s^{\nu}+\iota \geq 0, \forall(b, w) \in X \times U\right\} .
\end{aligned}
$$

Remark 3.4. Clearly, $\Theta=\Theta_{\iota}$, when $\iota=0$ and $\Theta \subseteq \Theta_{\iota}, \forall \iota>0$.

Further, for a set $B$, the symbol diam $B$ stands for the diameter of $B$, and it is defined as follows

$$
\operatorname{diam} B=\sup _{\alpha, \beta \in B}\|\alpha-\beta\| .
$$

Now, we are able to formulate and prove a first characterization result on the well-posedness of (CVIP).

Theorem 3.5. Assume the curvilinear integral functional $F$ is monotone and hemicontinuous on $X \times U$. Then the problem (CVIP) is well-posed if and only if

$$
\Theta_{\iota} \neq \emptyset, \forall \iota>0 \text { and } \operatorname{diam} \Theta_{\iota} \rightarrow 0 \text { as } \iota \rightarrow 0 .
$$

Proof. Suppose the problem (CVIP) is well-posed. Then it possesses a single solution $(\bar{a}, \bar{u}) \in \Theta$. Since $\Theta \subseteq$ $\Theta_{\iota}, \forall \iota>0$, therefore, $\Theta_{\iota} \neq \emptyset, \forall \iota>0$. Suppose, contrary to the result, that diam $\Theta_{\iota} \nrightarrow 0$ as $\iota \rightarrow 0$. Then there exist $r>0$, a positive integer $m$, a sequence of real numbers $\iota_{n}>0$ with $\iota_{n} \rightarrow 0$, and two elements $\left(a_{n}, u_{n}\right)$ and $\left(a_{n}^{\prime}, u_{n}^{\prime}\right) \in \Theta_{\iota_{n}}$ such that

$$
\left\|\left(a_{n}(s), u_{n}(s)\right)-\left(a_{n}^{\prime}(s), u_{n}^{\prime}(s)\right)\right\|>r, \quad \forall n \geq m .
$$

Since $\left(a_{n}, u_{n}\right),\left(a_{n}^{\prime}, u_{n}^{\prime}\right) \in \Theta_{\iota_{n}}$, we get

$$
\int_{\Upsilon}\left[\left(b(s)-a_{n}(s)\right) \frac{\partial f_{\nu}}{\partial a}\left(\psi_{a_{n}, u_{n}}(s)\right)+\left(w(s)-u_{n}(s)\right) \frac{\partial f_{\nu}}{\partial u}\left(\psi_{a_{n}, u_{n}}(s)\right)\right.
$$




$$
\left.+D_{\kappa}\left(b(s)-a_{n}(s)\right) \frac{\partial f_{\nu}}{\partial a_{\kappa}}\left(\psi_{a_{n}, u_{n}}(s)\right)\right] \mathrm{d} s^{\nu}+\iota_{n} \geq 0, \quad \forall(b, w) \in X \times U
$$

and

$$
\begin{aligned}
& \int_{\Upsilon}\left[\left(b(s)-a_{n}^{\prime}(s)\right) \frac{\partial f_{\nu}}{\partial a}\left(\psi_{a_{n}^{\prime}, u_{n}^{\prime}}(s)\right)+\left(w(s)-u_{n}^{\prime}(s)\right) \frac{\partial f_{\nu}}{\partial u}\left(\psi_{a_{n}^{\prime}, u_{n}^{\prime}}(s)\right)\right. \\
+ & \left.D_{\kappa}\left(b(s)-a_{n}^{\prime}(s)\right) \frac{\partial f_{\nu}}{\partial a_{\kappa}}\left(\psi_{a_{n}^{\prime}, u_{n}^{\prime}}(s)\right)\right] \mathrm{d} s^{\nu}+\iota_{n} \geq 0, \quad \forall(b, w) \in X \times U .
\end{aligned}
$$

Clearly, it follows that $\left\{\left(a_{n}, u_{n}\right)\right\}$ and $\left\{\left(a_{n}^{\prime}, u_{n}^{\prime}\right)\right\}$ are approximating sequences of (CVIP) which converge to $(\bar{a}, \bar{u})$ since, by hypothesis, the problem (CVIP) is well-posed. By direct computation, we get

$$
\begin{gathered}
\left\|\left(a_{n}(s), u_{n}(s)\right)-\left(a_{n}^{\prime}(s), u_{n}^{\prime}(s)\right)\right\| \\
=\left\|\left(a_{n}(s), u_{n}(s)\right)-(\bar{a}(s), \bar{u}(s))+(\bar{a}(s), \bar{u}(s))-\left(a_{n}^{\prime}(s), u_{n}^{\prime}(s)\right)\right\| \\
\leq\left\|\left(a_{n}(s), u_{n}(s)\right)-(\bar{a}(s), \bar{u}(s))\right\|+\left\|(\bar{a}(s), \bar{u}(s))-\left(a_{n}^{\prime}(s), u_{n}^{\prime}(s)\right)\right\| \leq \iota,
\end{gathered}
$$

which contradicts (1), for some $\iota=r$.

Conversely, consider $\left\{\left(a_{n}, u_{n}\right)\right\}$ is an approximating sequence of (CVIP). Then there exists a sequence of positive real numbers $\iota_{n} \rightarrow 0$ as $n \rightarrow \infty$ such that

$$
\begin{aligned}
& \int_{\Upsilon}\left[\left(b(s)-a_{n}(s)\right) \frac{\partial f_{\nu}}{\partial a}\left(\psi_{a_{n}, u_{n}}(s)\right)+\left(w(s)-u_{n}(s)\right) \frac{\partial f_{\nu}}{\partial u}\left(\psi_{a_{n}, u_{n}}(s)\right)\right. \\
& \left.+D_{\kappa}\left(b(s)-a_{n}(s)\right) \frac{\partial f_{\nu}}{\partial a_{\kappa}}\left(\psi_{a_{n}, u_{n}}(s)\right)\right] \mathrm{d} s^{\nu}+\iota_{n} \geq 0, \quad \forall(b, w) \in X \times U
\end{aligned}
$$

holds, involving that $\left(a_{n}, u_{n}\right) \in \Theta_{\iota_{n}}$. Since diam $\Theta_{\iota_{n}} \rightarrow 0$ as $\iota_{n} \rightarrow 0$, therefore $\left\{\left(a_{n}, u_{n}\right)\right\}$ is a Cauchy sequence which converges to some $(\bar{a}, \bar{u}) \in X \times U$ as $X \times U$ is a closed set.

By hypothesis, the curvilinear integral functional $\int_{\Upsilon} f_{\nu}\left(\psi_{a, u}(s)\right) \mathrm{d} s^{\nu}$ is monotone on $X \times U$. Therefore, by Definition 2.1, for $(\bar{a}, \bar{u}),(b, w) \in X \times U$, we have

$$
\begin{gathered}
\int_{\Upsilon}\left[(\bar{a}(s)-b(s))\left(\frac{\partial f_{\nu}}{\partial a}\left(\psi_{\bar{a}, \bar{u}}(s)\right)-\frac{\partial f_{\nu}}{\partial a}\left(\psi_{b, w}(s)\right)\right)\right. \\
+(\bar{u}(s)-w(s))\left(\frac{\partial f_{\nu}}{\partial u}\left(\psi_{\bar{a}, \bar{u}}(s)\right)-\frac{\partial f_{\nu}}{\partial a}\left(\psi_{b, w}(s)\right)\right) \\
\left.+D_{\kappa}(\bar{a}(s)-b(s))\left(\frac{\partial f_{\nu}}{\partial a_{\kappa}}\left(\psi_{\bar{a}, \bar{u}}(s)\right)-\frac{\partial f_{\nu}}{\partial a_{\kappa}}\left(\psi_{b, w}(s)\right)\right)\right] \mathrm{d} s^{\nu} \geq 0
\end{gathered}
$$

or, equivalently,

$$
\begin{gathered}
\int_{\Upsilon}\left[(\bar{a}(s)-b(s)) \frac{\partial f_{\nu}}{\partial a}\left(\psi_{\bar{a}, \bar{u}}(s)\right)+(\bar{u}(s)-w(s)) \frac{\partial f_{\nu}}{\partial u}\left(\psi_{\bar{a}, \bar{u}}(s)\right)\right. \\
\left.+D_{\kappa}(\bar{a}(s)-b(s)) \frac{\partial f_{\nu}}{\partial a_{\kappa}}\left(\psi_{\bar{a}, \bar{u}}(s)\right)\right] \mathrm{d} s^{\nu} \\
\geq \int_{\Upsilon}\left[(\bar{a}(s)-b(s)) \frac{\partial f_{\nu}}{\partial a}\left(\psi_{b, w}(s)\right)+(\bar{u}(s)-w(s)) \frac{\partial f_{\nu}}{\partial u}\left(\psi_{b, w}(s)\right)\right.
\end{gathered}
$$




$$
\left.+D_{\kappa}(\bar{a}(s)-b(s)) \frac{\partial f_{\nu}}{\partial a_{\kappa}}\left(\psi_{b, w}(s)\right)\right] \mathrm{d} s^{\nu} .
$$

Taking limit in inequality (3.2), we have

$$
\begin{gathered}
\int_{\Upsilon}\left[(\bar{a}(s)-b(s)) \frac{\partial f_{\nu}}{\partial a}\left(\psi_{\bar{a}, \bar{u}}(s)\right)+(\bar{u}(s)-w(s)) \frac{\partial f_{\nu}}{\partial u}\left(\psi_{\bar{a}, \bar{u}}(s)\right)\right. \\
\left.+D_{\kappa}(\bar{a}(s)-b(s)) \frac{\partial f_{\nu}}{\partial a_{\kappa}}\left(\psi_{\bar{a}, \bar{u}}(s)\right)\right] \mathrm{d} s^{\nu} \leq 0 .
\end{gathered}
$$

On combining (3.3) and (3.4), we obtain

$$
\begin{gathered}
\int_{\Upsilon}\left[(b(s)-\bar{a}(s)) \frac{\partial f_{\nu}}{\partial a}\left(\psi_{b, w}(s)\right)+(w(s)-\bar{u}(s)) \frac{\partial f_{\nu}}{\partial u}\left(\psi_{b, w}(s)\right)\right. \\
\left.+D_{\kappa}(b(s)-\bar{a}(s)) \frac{\partial f_{\nu}}{\partial a_{\kappa}}\left(\psi_{b, w}(s)\right)\right] \mathrm{d} s^{\nu} \geq 0 .
\end{gathered}
$$

Further, taking into account Lemma 2.6, it follows

$$
\begin{gathered}
\int_{\Upsilon}\left[(b(s)-\bar{a}(s)) \frac{\partial f_{\nu}}{\partial a}\left(\psi_{\bar{a}, \bar{u}}(s)\right)+(w(s)-\bar{u}(s)) \frac{\partial f_{\nu}}{\partial u}\left(\psi_{\bar{a}, \bar{u}}(s)\right)\right. \\
\left.+D_{\kappa}(b(s)-\bar{a}(s)) \frac{\partial f_{\nu}}{\partial a_{\kappa}}\left(\psi_{\bar{a}, \bar{u}}(s)\right)\right] \mathrm{d} s^{\nu} \geq 0,
\end{gathered}
$$

which implies that $(\bar{a}, \bar{u}) \in \Theta$. It remains to prove that $(\bar{a}, \bar{u})$ is a single solution of (CVIP). Contrarily, suppose $\left(a_{1}, u_{1}\right),\left(a_{2}, u_{2}\right)$ be two distinct solutions of (CVIP). Then

$$
0<\left\|\left(a_{1}(s), u_{1}(s)\right)-\left(a_{2}(s), u_{2}(s)\right)\right\| \leq \operatorname{diam} \Theta_{\iota} \rightarrow 0 \text { as } \iota \rightarrow 0
$$

which is not possible, and the proof is now complete.

Corollary 3.6. Assume that all the assumptions of Theorem 3.5 hold. Then the problem (CVIP) is well-posed if and only if

$$
\Theta \neq \emptyset \text { and } \operatorname{diam} \Theta_{\iota} \rightarrow 0 \text { as } \iota \rightarrow 0 \text {. }
$$

Proof. The proof follows the similar lines of Theorem 3.5, hence it is omitted.

In the next theorem, we establish that the well-posedness of (CVIP) is equivalent to the existence and uniqueness of solution.

Theorem 3.7. Assume the curvilinear integral functional $F$ is monotone and hemicontinuous on $X \times U$. Then (CVIP) is well-posed if and only if it possesses a single solution.

Proof. Assume that the problem (CVIP) is well-posed. In consequence, it possesses a single solution $\left(a_{0}, u_{0}\right)$. Conversely, suppose that (CVIP) has a single solution $\left(a_{0}, u_{0}\right)$ but it is not well-posed. Then, there exists an approximating sequence $\left\{\left(a_{n}, u_{n}\right)\right\}$ of (CVIP) which does not converge to $\left(a_{0}, u_{0}\right)$. Since $\left\{\left(a_{n}, u_{n}\right)\right\}$ is an approximating sequence of (CVIP), there must exist a sequence of positive real numbers $\iota_{n} \rightarrow 0$ as $n \rightarrow \infty$ such that

$$
\int_{\Upsilon}\left[\left(b(s)-a_{n}(s)\right) \frac{\partial f_{\nu}}{\partial a}\left(\psi_{a_{n}, u_{n}}(s)\right)+\left(w(s)-u_{n}(s)\right) \frac{\partial f_{\nu}}{\partial u}\left(\psi_{a_{n}, u_{n}}(s)\right)\right.
$$




$$
\left.+D_{\kappa}\left(b(s)-a_{n}(s)\right) \frac{\partial f_{\nu}}{\partial a_{\kappa}}\left(\psi_{a_{n}, u_{n}}(s)\right)\right] \mathrm{d} s^{\nu}+\iota_{n} \geq 0, \quad \forall(b, w) \in X \times U
$$

Further, in order to prove the boundedness of $\left\{\left(a_{n}, u_{n}\right)\right\}$, we start by reductio ad absurdum. Suppose $\left\{\left(a_{n}, u_{n}\right)\right\}$ is not bounded, that is, $\left\|\left(a_{n}(s), u_{n}(s)\right)\right\| \rightarrow+\infty$ as $n \rightarrow+\infty$. Consider $\delta_{n}(s)=\frac{1}{\left\|\left(a_{n}(s), u_{n}(s)\right)-\left(a_{0}(s), u_{0}(s)\right)\right\|}$ and $\left(\mathrm{a}_{n}, \mathrm{u}_{n}\right)=\left(a_{0}, u_{0}\right)+\delta_{n}\left[\left(a_{n}, u_{n}\right)-\left(a_{0}, u_{0}\right)\right]$.

We can see that $\left\{\left(\mathrm{a}_{n}, \mathrm{u}_{n}\right)\right\}$ is bounded in $X \times U$. So, passing to a subsequence if necessary, we may assume that

$$
\left(\mathrm{a}_{n}, \mathrm{u}_{n}\right) \rightarrow(\mathrm{a}, \mathrm{u}) \text { weakly in } X \times U \neq\left\{\left(a_{0}, u_{0}\right)\right\}
$$

It is not difficult to verify that $(\mathrm{a}, \mathrm{u}) \neq\left(a_{0}, u_{0}\right)$ thanks to $\| \delta_{n}(s)\left[\left(a_{n}(s), u_{n}(s)\right)-\left(a_{0}(s), u_{0}(s)\right) \|=1\right.$ for all $n \in \mathbb{N}$. Since $\left(a_{0}, u_{0}\right)$ is solution of (CVIP), therefore

$$
\begin{gathered}
\int_{\Upsilon}\left[\left(b(s)-a_{0}(s)\right) \frac{\partial f_{\nu}}{\partial a}\left(\psi_{a_{0}, u_{0}}(s)\right)+\left(w(s)-u_{0}(s)\right) \frac{\partial f_{\nu}}{\partial u}\left(\psi_{a_{0}, u_{0}}(s)\right)\right. \\
\left.+D_{\kappa}\left(b(s)-a_{0}(s)\right) \frac{\partial f_{\nu}}{\partial a_{\kappa}}\left(\psi_{a_{0}, u_{0}}(s)\right)\right] \mathrm{d} s^{\nu} \geq 0, \quad \forall(b, w) \in X \times U .
\end{gathered}
$$

Thus, by Lemma 2.6, the above inequality implies that

$$
\begin{aligned}
& \int_{\Upsilon}\left[\left(b(s)-a_{0}(s)\right) \frac{\partial f_{\nu}}{\partial a}\left(\psi_{b, w}(s)\right)+\left(w(s)-u_{0}(s)\right) \frac{\partial f_{\nu}}{\partial u}\left(\psi_{b, w}(s)\right)\right. \\
& \left.+D_{\kappa}\left(b(s)-a_{0}(s)\right) \frac{\partial f_{\nu}}{\partial a_{\kappa}}\left(\psi_{b, w}(s)\right)\right] \mathrm{d} s^{\nu} \geq 0, \quad \forall(b, w) \in X \times U
\end{aligned}
$$

By hypothesis, the curvilinear integral functional $F$ is monotone on $X \times U$, therefore, for $\left(a_{n}, u_{n}\right),(b, w) \in X \times U$, we have

$$
\begin{gathered}
\int_{\Upsilon}\left[\left(a_{n}(s)-b(s)\right)\left(\frac{\partial f_{\nu}}{\partial a}\left(\psi_{a_{n}, u_{n}}(s)\right)-\frac{\partial f_{\nu}}{\partial a}\left(\psi_{b, w}(s)\right)\right)\right. \\
+\left(u_{n}(s)-w(s)\right)\left(\frac{\partial f_{\nu}}{\partial u}\left(\psi_{a_{n}, u_{n}}(s)\right)-\frac{\partial f_{\nu}}{\partial a}\left(\psi_{b, w}(s)\right)\right) \\
\left.+D_{\kappa}\left(a_{n}(s)-b(s)\right)\left(\frac{\partial f_{\nu}}{\partial a_{\kappa}}\left(\psi_{a_{n}, u_{n}}(s)\right)-\frac{\partial f_{\nu}}{\partial a_{\kappa}}\left(\psi_{b, w}(s)\right)\right)\right] \mathrm{d} s^{\nu} \geq 0,
\end{gathered}
$$

or, equivalently,

$$
\begin{gathered}
\int_{\Upsilon}\left[\left(b(s)-a_{n}(s)\right) \frac{\partial f_{\nu}}{\partial a}\left(\psi_{a_{n}, u_{n}}(s)\right)+\left(w(s)-u_{n}(s)\right) \frac{\partial f_{\nu}}{\partial u}\left(\psi_{a_{n}, u_{n}}(s)\right)\right. \\
\left.+D_{\kappa}\left(b(s)-a_{n}(s)\right) \frac{\partial f_{\nu}}{\partial a_{\kappa}}\left(\psi_{a_{n}, u_{n}}(s)\right)\right] \mathrm{d} s^{\nu} \\
\leq \int_{\Upsilon}\left[\left(b(s)-a_{n}(s)\right) \frac{\partial f_{\nu}}{\partial a}\left(\psi_{b, w}(s)\right)+\left(w(s)-u_{n}(s)\right) \frac{\partial f_{\nu}}{\partial u}\left(\psi_{b, w}(s)\right)\right. \\
\left.+D_{\kappa}\left(b(s)-a_{n}(s)\right) \frac{\partial f_{\nu}}{\partial a_{\kappa}}\left(\psi_{b, w}(s)\right)\right] \mathrm{d} s^{\nu}
\end{gathered}
$$


Combining with (3.5) and (3.7), we have

$$
\begin{aligned}
& \int_{\Upsilon}\left[\left(b(s)-a_{n}(s)\right) \frac{\partial f_{\nu}}{\partial a}\left(\psi_{b, w}(s)\right)+\left(w(s)-u_{n}(s)\right) \frac{\partial f_{\nu}}{\partial u}\left(\psi_{b, w}(s)\right)\right. \\
& \left.+D_{\kappa}\left(b(s)-a_{n}(s)\right) \frac{\partial f_{\nu}}{\partial a_{\kappa}}\left(\psi_{b, w}(s)\right)\right] \mathrm{d} s^{\nu} \geq-\iota_{n}, \quad \forall(b, w) \in X \times U .
\end{aligned}
$$

Because of $\delta_{n} \rightarrow 0$ as $n \rightarrow \infty$ (by the assumption that $\left\{\left(a_{n}, u_{n}\right)\right\}$ is not bounded), so, we can take $n_{0} \in \mathbb{N}$ be large enough such that $\delta_{n}<1$ for all $n \geq n_{0}$. Multiplying the inequality above and (3.6) by $\delta_{n}(s)>0$ and $1-\delta_{n}(s)>0$, respectively, we sum the resulting inequalities to get

$$
\begin{gathered}
\int_{\Upsilon}\left[\left(b(s)-\mathrm{a}_{n}(s)\right) \frac{\partial f_{\nu}}{\partial a}\left(\psi_{b, w}(s)\right)+\left(w(s)-\mathrm{u}_{n}(s)\right) \frac{\partial f_{\nu}}{\partial u}\left(\psi_{b, w}(s)\right)\right. \\
\left.+D_{\kappa}\left(b(s)-\mathrm{a}_{n}(s)\right) \frac{\partial f_{\nu}}{\partial a_{\kappa}}\left(\psi_{b, w}(s)\right)\right] \mathrm{d} s^{\nu} \geq-\iota_{n}, \quad \forall(b, w) \in X \times U, \forall n \geq n_{0} .
\end{gathered}
$$

Since $\left(\mathrm{a}_{n}, \mathrm{u}_{n}\right) \rightarrow(\mathrm{a}, \mathrm{u}) \neq\left(a_{0}, u_{0}\right)$ and $\left(\mathrm{a}_{n}, \mathbf{u}_{n}\right)=\left(a_{0}, u_{0}\right)+\left(\mathbf{a}_{n}, \mathbf{u}_{n}\right)\left[\left(a_{n}, u_{n}\right)-\left(a_{0}, u_{0}\right)\right]$, we have

$$
\begin{gathered}
\int_{\Upsilon}\left[(b(s)-\mathrm{a}(s)) \frac{\partial f_{\nu}}{\partial a}\left(\psi_{b, w}(s)\right)+(w(s)-\mathrm{u}(s)) \frac{\partial f_{\nu}}{\partial u}\left(\psi_{b, w}(s)\right)\right. \\
\left.+D_{\kappa}(b(s)-\mathrm{a}(s)) \frac{\partial f_{\nu}}{\partial a_{\kappa}}\left(\psi_{b, w}(s)\right)\right] \mathrm{d} s^{\nu} \\
=\lim _{n \rightarrow \infty} \int_{\Upsilon}\left[\left(b(s)-\mathrm{a}_{n}(s)\right) \frac{\partial f_{\nu}}{\partial a}\left(\psi_{b, w}(s)\right)+\left(w(s)-\mathrm{u}_{n}(s)\right) \frac{\partial f_{\nu}}{\partial u}\left(\psi_{b, w}(s)\right)\right. \\
\left.+D_{\kappa}\left(b(s)-\mathrm{a}_{n}(s)\right) \frac{\partial f_{\nu}}{\partial a_{\kappa}}\left(\psi_{b, w}(s)\right)\right] \mathrm{d} s^{\nu} \\
\geq-\lim _{n \rightarrow \infty} \iota_{n}=0, \quad \forall(b, w) \in X \times U .
\end{gathered}
$$

Thus, by Lemma 2.6, we have

$$
\begin{aligned}
& \int_{\Upsilon}\left[(b(s)-\mathrm{a}(s)) \frac{\partial f_{\nu}}{\partial a}\left(\psi_{\mathrm{a}, \mathrm{u}}(s)\right)+(w(s)-\mathrm{u}(s)) \frac{\partial f_{\nu}}{\partial u}\left(\psi_{\mathrm{a}, \mathrm{u}}(s)\right)\right. \\
& \left.+D_{\kappa}(b(s)-\mathrm{a}(s)) \frac{\partial f_{\nu}}{\partial a_{\kappa}}\left(\psi_{\mathrm{a}, \mathrm{u}}(s)\right)\right] \mathrm{d} s^{\nu} \geq 0, \quad \forall(b, w) \in X \times U .
\end{aligned}
$$

This implies that $(\mathrm{a}, \mathrm{u})$ is solution of (CVIP), which contradicts the uniqueness of $\left(a_{0}, u_{0}\right)$. Therefore, $\left\{\left(a_{n}, u_{n}\right)\right\}$ is a bounded sequence having convergent subsequence $\left\{\left(a_{n_{k}}, u_{n_{k}}\right)\right\}$ which converges to $(\bar{a}, \bar{u}) \in X \times U$ as $k \rightarrow \infty$. Again, from the definition of monotonicity, for $\left(a_{n_{k}}, u_{n_{k}}\right),(b, w) \in X \times U$, we have (see (3.7))

$$
\begin{gathered}
\int_{\Upsilon}\left[\left(b(s)-a_{n_{k}}(s)\right) \frac{\partial f_{\nu}}{\partial a}\left(\psi_{a_{n_{k}}, u_{n_{k}}}(s)\right)+\left(w(s)-u_{n_{k}}(s)\right) \frac{\partial f_{\nu}}{\partial u}\left(\psi_{a_{n_{k}}, u_{n_{k}}}(s)\right)\right. \\
\left.+D_{\kappa}\left(b(s)-a_{n_{k}}(s)\right) \frac{\partial f_{\nu}}{\partial a_{\kappa}}\left(\psi_{a_{n_{k}}, u_{n_{k}}}(s)\right)\right] \mathrm{d} s^{\nu} \\
\leq \int_{\Upsilon}\left[\left(b(s)-a_{n_{k}}(s)\right) \frac{\partial f_{\nu}}{\partial a}\left(\psi_{b, w}(s)\right)+\left(w(s)-u_{n_{k}}(s)\right) \frac{\partial f_{\nu}}{\partial u}\left(\psi_{b, w}(s)\right)\right.
\end{gathered}
$$




$$
\left.+D_{\kappa}\left(b(s)-a_{n_{k}}(s)\right) \frac{\partial f_{\nu}}{\partial a_{\kappa}}\left(\psi_{b, w}(s)\right)\right] \mathrm{d} s^{\nu}
$$

Also, on behalf of (3.5), we can write

$$
\begin{aligned}
\lim _{k \rightarrow \infty} \int_{\Upsilon}[(b(s) & \left.-a_{n_{k}}(s)\right) \frac{\partial f_{\nu}}{\partial a}\left(\psi_{a_{n_{k}}, u_{n_{k}}}(s)\right)+\left(w(s)-u_{n_{k}}(s)\right) \frac{\partial f_{\nu}}{\partial u}\left(\psi_{a_{n_{k}}, u_{n_{k}}}(s)\right) \\
& \left.+D_{\kappa}\left(b(s)-a_{n_{k}}(s)\right) \frac{\partial f_{\nu}}{\partial a_{\kappa}}\left(\psi_{a_{n_{k}}, u_{n_{k}}}(s)\right)\right] \mathrm{d} s^{\nu} \geq 0
\end{aligned}
$$

Combining (3.9) and (3.10), we have

$$
\begin{gathered}
\lim _{k \rightarrow \infty} \int_{\Upsilon}\left[\left(b(s)-a_{n_{k}}(s)\right) \frac{\partial f_{\nu}}{\partial a}\left(\psi_{b, w}(s)\right)+\left(w(s)-u_{n_{k}}(s)\right) \frac{\partial f_{\nu}}{\partial u}\left(\psi_{b, w}(s)\right)\right. \\
\left.+D_{\kappa}\left(b(s)-a_{n_{k}}(s)\right) \frac{\partial f_{\nu}}{\partial a_{\kappa}}\left(\psi_{b, w}(s)\right)\right] \mathrm{d} s^{\nu} \geq 0 \\
\Rightarrow \int_{\Upsilon}\left[(b(s)-\bar{a}(s)) \frac{\partial f_{\nu}}{\partial a}\left(\psi_{b, w}(s)\right)+(w(s)-\bar{u}(s)) \frac{\partial f_{\nu}}{\partial u}\left(\psi_{b, w}(s)\right)\right. \\
\left.+D_{\kappa}(b(s)-\bar{a}(s)) \frac{\partial f_{\nu}}{\partial a_{\kappa}}\left(\psi_{b, w}(s)\right)\right] \mathrm{d} s^{\nu} \geq 0
\end{gathered}
$$

Thus, by Lemma 2.6, the above inequality implies that

$$
\begin{gathered}
\int_{\Upsilon}\left[(b(s)-\bar{a}(s)) \frac{\partial f_{\nu}}{\partial a}\left(\psi_{\bar{a}, \bar{u}}(s)\right)+(w(s)-\bar{u}(s)) \frac{\partial f_{\nu}}{\partial u}\left(\psi_{\bar{a}, \bar{u}}(s)\right)\right. \\
\left.+D_{\kappa}(b(s)-\bar{a}(s)) \frac{\partial f_{\nu}}{\partial a_{\kappa}}\left(\psi_{\bar{a}, \bar{u}}(s)\right)\right] \mathrm{d} s^{\nu} \geq 0 .
\end{gathered}
$$

which shows that $(\bar{a}, \bar{u})$ is solution of (CVIP). Hence, $\left(a_{n_{k}}, u_{n_{k}}\right) \rightarrow(\bar{a}, \bar{u})$, that is, $\left(a_{n_{k}}, u_{n_{k}}\right) \rightarrow\left(a_{0}, u_{0}\right)$, involving $\left(a_{n}, u_{n}\right) \rightarrow\left(a_{0}, u_{0}\right)$ and the proof is complete.

Further, we provide an illustrative application of Theorem 3.7.

Example 3.8. Let $\nu \in\{1,2\}, \Omega=[0,1]^{2}$ and $\Omega \supset \Upsilon$ be a piecewise smooth curve joining the points $(0,0),(1,1)$ in $\Omega$. Consider

$$
f_{\nu}\left(\psi_{a, u}(s)\right) \mathrm{d} s^{\nu}=f_{1}\left(\psi_{a, u}(s)\right) \mathrm{d} s^{1}+f_{2}\left(\psi_{a, u}(s)\right) \mathrm{d} s^{2}=u^{2}(s) \mathrm{d} s^{1}+\left(e^{a(s)}-a(s)\right) \mathrm{d} s^{2} .
$$

(CVIP-1): Find $(a, u) \in X \times U$ so that

$$
\int_{\Upsilon}\left[2(w(s)-u(s)) u(s) \mathrm{d} s^{1}+(b(s)-a(s))\left(e^{a(s)}-1\right) \mathrm{d} s^{2}\right] \geq 0, \forall(b, w) \in X \times U .
$$

Clearly, $\Theta=\{(0,0)\}$. It can be easily verified that the functional $\int_{\Upsilon} f_{\nu}\left(\psi_{a, u}(s)\right) \mathrm{d} s^{\nu}$ is monotone and hemicontinuous on the nonempty, closed and convex set $X \times U=C^{1}(\Omega,[-10,10]) \times C(\Omega,[-10,10])$. Since all the hypotheses of Theorem 3.7 hold, the problem (CVIP-1) is well-posed. Further, $\Theta_{\iota}=\{(0,0)\}$ and consequently, $\Theta_{\iota} \neq \emptyset$ and diam $\Theta_{\iota} \rightarrow 0$ as $\iota \rightarrow 0$. Thus, by Theorem 3.5, the problem (CVIP-1) is well-posed. 
The next result establishes that the well-posedness in generalized sense of (CVIP) is equivalent to the nonemptiness of its solution set.

Theorem 3.9. Suppose the curvilinear integral functional $F$ is monotone and hemicontinuous on the nonempty compact set $X \times U$. Then $(C V I P)$ is well-posed in generalized sense if and only if the solution set $\Theta$ is nonempty.

Proof. Suppose (CVIP) is well-posed in generalized sense. Consequently, its solution set $\Theta$ is nonempty. Conversely, let $\left\{\left(a_{n}, u_{n}\right)\right\}$ be an approximating sequence of (CVIP). Then there exists a sequence of positive real numbers $\iota_{n} \rightarrow 0$ such that

$$
\begin{gathered}
\int_{\Upsilon}\left[\left(b(s)-a_{n}(s)\right) \frac{\partial f_{\nu}}{\partial a}\left(\psi_{a_{n}, u_{n}}(s)\right)+\left(w(s)-u_{n}(s)\right) \frac{\partial f_{\nu}}{\partial u}\left(\psi_{a_{n}, u_{n}}(s)\right)\right. \\
\left.+D_{\kappa}\left(b(s)-a_{n}(s)\right) \frac{\partial f_{\nu}}{\partial a_{\kappa}}\left(\psi_{a_{n}, u_{n}}(s)\right)\right] \mathrm{d} s^{\nu}+\iota_{n} \geq 0, \quad \forall(b, w) \in X \times U .
\end{gathered}
$$

By hypothesis, $X \times U$ is a compact set and, therefore, $\left\{\left(a_{n}, u_{n}\right)\right\}$ has a subsequence $\left\{\left(a_{n_{k}}, u_{n_{k}}\right)\right\}$ which converges to some pair $\left(a_{0}, u_{0}\right) \in X \times U$. Since the integral functional $F$ is monotone on $X \times U$, for $\left(a_{n_{k}}, u_{n_{k}}\right),(b, w) \in$ $X \times U$, we have

$$
\begin{gathered}
\int_{\Upsilon}\left[\left(b(s)-a_{n_{k}}(s)\right) \frac{\partial f_{\nu}}{\partial a}\left(\psi_{a_{n_{k}}, u_{n_{k}}}(s)\right)+\left(w(s)-u_{n_{k}}(s)\right) \frac{\partial f_{\nu}}{\partial u}\left(\psi_{a_{n_{k}}, u_{n_{k}}}(s)\right)\right. \\
\left.+D_{\kappa}\left(b(s)-a_{n_{k}}(s)\right) \frac{\partial f_{\nu}}{\partial a_{\kappa}}\left(\psi_{a_{n_{k}}, u_{n_{k}}}(s)\right)\right] \mathrm{d} s^{\nu} \\
\leq \int_{\Upsilon}\left[\left(b(s)-a_{n_{k}}(s)\right) \frac{\partial f_{\nu}}{\partial a}\left(\psi_{b, w}(s)\right)+\left(w(s)-u_{n_{k}}(s)\right) \frac{\partial f_{\nu}}{\partial u}\left(\psi_{b, w}(s)\right)\right. \\
\left.+D_{\kappa}\left(b(s)-a_{n_{k}}(s)\right) \frac{\partial f_{\nu}}{\partial a_{\kappa}}\left(\psi_{b, w}(s)\right)\right] \mathrm{d} s^{\nu} .
\end{gathered}
$$

Taking limit $k \rightarrow \infty$ in the above inequality, we have

$$
\begin{gathered}
\lim _{k \rightarrow \infty} \int_{\Upsilon}\left[\left(b(s)-a_{n_{k}}(s)\right) \frac{\partial f_{\nu}}{\partial a}\left(\psi_{a_{n_{k}}, u_{n_{k}}}(s)\right)+\left(w(s)-u_{n_{k}}(s)\right) \frac{\partial f_{\nu}}{\partial u}\left(\psi_{a_{n_{k}}, u_{n_{k}}}(s)\right)\right. \\
\left.+D_{\kappa}\left(b(s)-a_{n_{k}}(s)\right) \frac{\partial f_{\nu}}{\partial a_{\kappa}}\left(\psi_{a_{n_{k}}, u_{n_{k}}}(s)\right)\right] \mathrm{d} s^{\nu} \\
\leq \lim _{k \rightarrow \infty} \int_{\Upsilon}\left[\left(b(s)-a_{n_{k}}(s)\right) \frac{\partial f_{\nu}}{\partial a}\left(\psi_{b, w}(s)\right)+\left(w(s)-u_{n_{k}}(s)\right) \frac{\partial f_{\nu}}{\partial u}\left(\psi_{b, w}(s)\right)\right. \\
\left.+D_{\kappa}\left(b(s)-a_{n_{k}}(s)\right) \frac{\partial f_{\nu}}{\partial a_{\kappa}}\left(\psi_{b, w}(s)\right)\right] \mathrm{d} s^{\nu}
\end{gathered}
$$

Since $\left\{\left(a_{n_{k}}, u_{n_{k}}\right)\right\}$ is an approximating subsequence in $X \times U$, therefore, on behalf of (3.11), we have

$$
\begin{gathered}
\lim _{k \rightarrow \infty} \int_{\Upsilon}\left[\left(b(s)-a_{n_{k}}(s)\right) \frac{\partial f_{\nu}}{\partial a}\left(\psi_{a_{n_{k}}, u_{n_{k}}}(s)\right)+\left(w(s)-u_{n_{k}}(s)\right) \frac{\partial f_{\nu}}{\partial u}\left(\psi_{a_{n_{k}}, u_{n_{k}}}(s)\right)\right. \\
\left.+D_{\kappa}\left(b(s)-a_{n_{k}}(s)\right) \frac{\partial f_{\nu}}{\partial a_{\kappa}}\left(\psi_{a_{n_{k}}, u_{n_{k}}}(s)\right)\right] \mathrm{d} s^{\nu} \geq 0, \quad \forall(b, w) \in X \times U
\end{gathered}
$$


Combining (3.12) and (3.13), we get

$$
\begin{gathered}
\lim _{k \rightarrow \infty} \int_{\Upsilon}\left[\left(b(s)-a_{n_{k}}(s)\right) \frac{\partial f_{\nu}}{\partial a}\left(\psi_{b, w}(s)\right)+\left(w(s)-u_{n_{k}}(s)\right) \frac{\partial f_{\nu}}{\partial u}\left(\psi_{b, w}(s)\right)\right. \\
\left.+D_{\kappa}\left(b(s)-a_{n_{k}}(s)\right) \frac{\partial f_{\nu}}{\partial a_{\kappa}}\left(\psi_{b, w}(s)\right)\right] \mathrm{d} s^{\nu} \geq 0, \quad \forall(b, w) \in X \times U \\
\Rightarrow \int_{\Upsilon}\left[\left(b(s)-a_{0}(s)\right) \frac{\partial f_{\nu}}{\partial a}\left(\psi_{b, w}(s)\right)+\left(w(s)-u_{0}(s)\right) \frac{\partial f_{\nu}}{\partial u}\left(\psi_{b, w}(s)\right)\right. \\
\left.\quad+D_{\kappa}\left(b(s)-a_{0}(s)\right) \frac{\partial f_{\nu}}{\partial a_{\kappa}}\left(\psi_{b, w}(s)\right)\right] \mathrm{d} s^{\nu} \geq 0, \quad \forall(b, w) \in X \times U .
\end{gathered}
$$

Thus, by Lemma 2.6, we can also write

$$
\begin{aligned}
& \int_{\Upsilon}\left[\left(b(s)-a_{0}(s)\right) \frac{\partial f_{\nu}}{\partial a}\left(\psi_{a_{0}, u_{0}}(s)\right)+\left(w(s)-u_{0}(s)\right) \frac{\partial f_{\nu}}{\partial u}\left(\psi_{a_{0}, u_{0}}(s)\right)\right. \\
& \left.+D_{\kappa}\left(b(s)-a_{0}(s)\right) \frac{\partial f_{\nu}}{\partial a_{\kappa}}\left(\psi_{a_{0}, u_{0}}(s)\right)\right] \mathrm{d} s^{\nu} \geq 0, \quad \forall(b, w) \in X \times U .
\end{aligned}
$$

which shows that $\left(a_{0}, u_{0}\right) \in \Theta$ and the proof is complete.

In the following, we present the sufficiency of well-posedness in generalized sense of (CVIP).

Theorem 3.10. Suppose the curvilinear integral functional $F$ is monotone and hemicontinuous on the nonempty compact set $X \times U$. Then $(C V I P)$ is well-posed in generalized sense if there exists $\iota>0$ such that $\Theta_{\iota}$ is a nonempty bounded set.

Proof. Let $\iota>0$ such that $\Theta_{\iota}$ is nonempty and bounded. Suppose $\left\{\left(a_{n}, u_{n}\right)\right\}$ is an approximating sequence of (CVIP). Then there exists a sequence of positive real numbers $\iota_{n} \rightarrow 0$ such that

$$
\begin{aligned}
& \int_{\Upsilon}\left[\left(b(s)-a_{n}(s)\right) \frac{\partial f_{\nu}}{\partial a}\left(\psi_{a_{n}, u_{n}}(s)\right)+\left(w(s)-u_{n}(s)\right) \frac{\partial f_{\nu}}{\partial u}\left(\psi_{a_{n}, u_{n}}(s)\right)\right. \\
& \left.+D_{\kappa}\left(b(s)-a_{n}(s)\right) \frac{\partial f_{\nu}}{\partial a_{\kappa}}\left(\psi_{a_{n}, u_{n}}(s)\right)\right] \mathrm{d} s^{\nu}+\iota_{n} \geq 0, \quad \forall(b, w) \in X \times U
\end{aligned}
$$

holds, which implies that $\left(a_{n}, u_{n}\right) \in \Theta_{\iota}, \forall n>m$ (see $m$ as a positive integer depending on $\iota$ ). Therefore, $\left\{\left(a_{n}, u_{n}\right)\right\}$ is a bounded sequence having a convergent subsequence $\left\{\left(a_{n_{k}}, u_{n_{k}}\right)\right\}$ which weakly converges to $\left(a_{0}, u_{0}\right)$ as $k \rightarrow \infty$. Proceeding in the similar lines of the proof of Theorem 3.9, we get $\left(a_{0}, u_{0}\right) \in \Theta$ and the proof is complete.

\section{Conclusions}

In this paper, based on the new concepts of monotonicity, pseudomonotonicity and hemicontinuity associated with curvilinear integral functionals, we have studied well-posedness and well-posedness in generalized sense for a class of controlled variational inequality problems. More concretely, we have established that, under suitable conditions, the well-posedness is characterized in terms of existence and uniqueness of solution. Moreover, sufficient conditions were presented for the well-posedness in generalized sense by assuming the boundedness of approximating solution set. Also, several illustrative examples have been formulated throughout the paper. 


\section{REFERENCES}

[1] L.C. Ceng, H. Gupta and C.F. Wen, Well-posedness by perturbations of variational hemivariational inequalities with perturbations. Filomat 26 (2012) 881-895.

[2] L.C. Ceng, N. Hadjisavvas, S. Schaible and J.C. Yao, Well-posedness for mixed quasivariational-like inequalities. J. Optim. Theory Appl. 139 (2008) 109-125.

[3] L.C. Ceng and J.C. Yao, Well-posedness of generalized mixed variational inequalities, inclusion problems and fixed-point problems. Nonlinear Anal. 69 (2008) 4585-4603.

[4] J.W. Chen, Z. Wang and Y.J. Cho, Levitin-Polyak well-posedness by perturbations for systems of set-valued vector quasiequilibrium problems. Math. Meth. Oper. Res. 77 (2013) 33-64.

[5] Y.P. Fang and R. Hu, Parametric well-posedness for variational inequalities defined by bifunctions. Comput. Math. Appl. 53 (2007) 1306-1316.

[6] Y.P. Fang, R. Hu and N.J. Huang, Well-posedness for equilibrium problems and for optimization problems with equilibrium constraints. Comput. Math. Appl. 55 (2008) 89-100.

[7] Y.P. Fang, N.J. Huang and J.C. Yao, Well-posedness of mixed variational inequalities, inclusion problems and fixed point problems. J. Global Optim. 41 (2008) 117-133.

[8] Y.P. Fang, N.J. Huang and J.C. Yao, Well-posedness by perturbations of mixed variational inequalities in Banach spaces. Eur J. Oper. Res. 201 (2010) 682-692.

[9] D. Goeleven and D. Mentagui, Well-posed hemivariational inequalities. Numer. Funct. Anal. Optim. 16 (1995) $909-921$.

[10] P.M.H. Heemels, M.K.C. Camlibel, A.J. Vander Schaft and J.M. Schumacher, Well-posedness of the complementarity class of hybrid systems, in Proc. IFAC 15th Triennial World Congress, Barcelona, Spain (2002).

[11] R. Hu, Y.B. Xiao, N.J. Huang and X. Wang, Equivalence results of well-posedness for split variational-hemivariational inequalities. J. Nonlinear Convex Anal. 20 (2019) 447-459.

[12] X.X. Huang, X.Q. Yang and D.L. Zhu, Levitin-Polyak well-posedness of variational inequality problems with functional constraints. J. Global Optim. 44 (2009) 159-174.

[13] A. Jayswal and S. Jha, Well-posedness for generalized mixed vector variational-like inequality problems in Banach space. Math. Commun. 22 (2017) 287-302.

[14] C.S. Lalitha and G. Bhatia, Well-posedness for variational inequality problems with generalized monotone set-valued maps. Numer. Funct. Anal. Optim. 30 (2009) 548-565.

[15] C.S. Lalitha and G. Bhatia, Well-posedness for parametric quasivariational inequality problems and for optimization problems with quasivariational inequality constraints. Optimization 59 (2010) 997-1011.

[16] E.S. Levitin and B.T. Polyak, Convergence of minimizing sequences in conditional extremum problems. Sov. Math. Dokl. 7 (1996) 764-767.

[17] M.B. Lignola, Well-posedness and L-well-posedness for quasivariational inequalities. J. Optim. Theory Appl. 128 (2006) $119-138$.

[18] M.B. Lignola and J. Morgan, Well-posedness for optimization problems with constraints defined by variational inequalities having a unique solution. J. Global Optim. 16 (2000) 57-67.

[19] M.B. Lignola and J. Morgan, $\alpha$-Well-posedness for Nash equilibria and for optimization problems with Nash equilibrium constraints. J. Global Optim. 36 (2006) 439-459.

[20] L.J. Lin and C.S. Chuang, Well-posedness in the generalized sense for variational inclusion and disclusion problems and well-posedness for optimization problems with constraint. Nonlinear Anal. 70 (2009) 3609-3617.

[21] Q.Y. Shu, R. Hu and Y.B. Xiao, Metric characterizations for well-psedness of split hemivariational inequalities. J. Inequal. Appl. 190 (2018) 2018.

[22] S. Treanţă, A necessary and sufficient condition of optimality for a class of multidimensional control problems. Optim. Control Appl. Meth. 41 (2020) 2137-2148.

[23] S. Treanţă and Şt. Mititelu, Efficiency for variational control problems on Riemann manifolds with geodesic quasiinvex curvilinear integral functionals, Revista de la Real Academia de Ciencias Exactas, Físicas y Naturales. Serie A. Matemáticas 14 (2020) 113.

[24] S. Treanţă, M. Arana-Jiménez and T. Antczak, A necessary and sufficient condition on the equivalence between local and global optimal solutions in variational control problems. Nonlinear Anal. 191 (2020) UNSP 111640.

[25] S. Treanță, On a modified optimal control problem with first-order PDE constraints and the associated saddle-point optimality criterion. Eur. J. Control 51 (2020) 1-9.

[26] S. Treanță, Efficiency in generalized V-KT-pseudoinvex control problems. Int. J. Control 93 (2020) 611-618.

[27] S. Treanţă and S. Singh, Weak sharp solutions associated with a multidimensional variational-type inequality. Positivity 25 (2021) 329-351.

[28] S. Treanţă, Some results on $(\rho, b, d)$-variational inequalities. J. Math. Ineq. 14 (2020) 805-818.

[29] S. Treanţă, On weak sharp solutions in $(\rho, b, d)$-variational inequalities. J. Ineq. Appl. 2020 (2020) 54.

[30] A.N. Tykhonov, On the stability of the functional optimization. USSR Comput. Math. Math. Phys. 6 (1966) 631-634.

[31] F. Usman and S.A. Khan, A generalized mixed vector variational-like inequality problem. Nonlinear Anal. 71 (2009) 5354-5362.

[32] G. Virmani and M. Srivastava, Various types of well-posedness for mixed vector quasivariational-like inequality using bifunctions. J. Appl. Math. Inform. 32 (2014) 427-439. 
[33] Y.M. Wang, Y.B. Xiao, X. Wang and Y.J. Cho, Equivalence of well-posedness between systems of hemivariational inequalities and inclusion problems. J. Nonlinear Sci. Appl. 9 (2016) 1178-1192.

[34] Y.B. Xiao, N.J. Huang and M.M. Wong, Well-posedness of hemivariational inequalities and inclusion problems. Taiwanese J. Math. 15 (2011) 1261-1276.

\section{Subscribe to Open (S20) A fair and sustainable open access model}

This journal is currently published in open access under a Subscribe-to-Open model (S2O). S2O is a transformative model that aims to move subscription journals to open access. Open access is the free, immediate, online availability of research articles combined with the rights to use these articles fully in the digital environment. We are thankful to our subscribers and sponsors for making it possible to publish this journal in open access, free of charge for authors.

\section{Please help to maintain this journal in open access!}

Check that your library subscribes to the journal, or make a personal donation to the $\mathrm{S} 2 \mathrm{O}$ programme, by contacting subscribers@edpsciences.org

More information, including a list of sponsors and a financial transparency report, available at: https://www.edpsciences.org/en/maths-s2o-programme 